\title{
Supplemental oxygen and nasal intermittent positive pressure ventilation
}

\author{
A.J. Padkin*, W.J.M. Kinnear**
}

Supplemental oxygen and nasal intermittent positive pressure ventilation. A.J. Padkin, W.J.M. Kinnear. CERS Journals Ltd 1996.

ABSTRACT: When supplementary oxygen is necessary with nasal intermittent positive pressure ventilation (NIPPV), the optimal route by which it should be added to the ventilator circuit is unknown. We investigated the oxygen concentration received when oxygen was supplied at flow rates between 0 and $6 \mathrm{~L} \cdot \mathrm{min}^{-1}$ into the proximal ventilator tubing or the nasal mask whilst patients were ventilated with air.

Eleven patients with stable chronic hypercapnic respiratory failure were studied. A calibration curve was produced for each by supplying different known oxygen concentrations through a Monnal D or DCC ventilator and measuring the arterial oxygen saturations achieved. Oxygen was then supplied into the ventilator tubing or nasal mask and arterial saturation again measured. The oxygen concentration received was estimated using the calibration curve. Tracheal oxygen concentration throughout the respiratory cycle was studied in one patient when oxygen was supplied by both routes.

Peak inspired oxygen concentration occurred at end-inspiration when oxygen was supplied into the ventilator tubing, but at mid-inspiration when supplied into the nasal mask. However, there was no significant difference between the two routes in the inspired oxygen concentration achieved at all flow rates: $1 \mathrm{~L} \cdot \mathrm{min}^{-1}$ supplied approximately $31 \%$ oxygen; $2 \mathrm{~L} \cdot \mathrm{min}^{-1} 37 \% ; 3 \mathrm{~L} \cdot \mathrm{min}^{-1} 40 \%$; and $4 \mathrm{~L} \cdot \mathrm{min}^{-1} 44 \%$. Flow rates above $4 \mathrm{~L} \cdot \mathrm{min}^{-1}$ had little additional effect.

In conclusion, oxygen supplementation during nasal intermittent positive pressure ventilation can be provided into the ventilator tubing or the nasal mask with equal efficiency.

Eur Respir J., 1996, 9, 834-836.

Nasal intermittent positive pressure ventilation (NIPPV) is increasingly being used in the treatment both of acute and chronic hypercapnic respiratory failure. Patients with chronic respiratory failure who are treated with long-term nocturnal NIPPV are usually adequately oxygenated with air as the inspired gas, although a small minority need supplemental oxygen [1]. However, supplemental oxygen is needed much more frequently when NIPPV is used in the treatment of acute respiratory failure [2].

Some of the more sophisticated ventilators used for NIPPV allow oxygen to be delivered through the ventilator, which then delivers a constant oxygen concentration throughout inspiration. Others do not have this facility, and supplemental oxygen must be added into the ventilator tubing or into a port on the nasal mask.

When supplemental oxygen is added to either the ventilator tubing or nasal mask, the oxygen concentration supplied to the patient is unknown. This study investigated the oxygen concentration received by the patient when oxygen was given at different flow rates by each of these routes.

\section{Patients and methods}

Eleven patients (4 females and 7 males) with stable, chronic, hypercapnic respiratory failure due to chest wall
Depts of *Anaesthesia and **Respiratory Medicine, University Hospital, Queen's Medical Centre, Nottingham, UK.

Correspondence: W.J.M. Kinnear Dept of Respiratory Medicine University Hospital

Queen's Medical Centre

Nottingham NG7 2UH

UK

\section{Keywords:}

Intermittent positive pressure ventilation oxygen inhalation therapy

Received: June 291995

Accepted after revision December 261995 disease were studied. Each was already receiving nocturnal NIPPV at home. They were all investigated using a Monnal D or DCC ventilator (Deva Medical Electronics) with standard ventilator tubing (Deva Medical Electronics) of approximately $620 \mathrm{~mL}$ internal volume and a nasal mask (Respironics Silicone Contour Mask 9037) of approximately $120 \mathrm{~mL}$ internal volume. Ventilator frequency, inspiratory flow rate and inspiratory:expiratory ratio were adjusted for each patient individually until they felt most comfortable on the ventilator.

Oxygen was initially supplied at flow rates between 0 and $6 \mathrm{~L} \cdot \mathrm{min}^{-1}$ through the ventilator (fig. 1a) with inspired oxygen concentration measured using a galvanic fuel cell oxygen analyser (Hudson 5524). After $5 \mathrm{~min}$ at each flow rate, the patient's oxygen saturation was measured with finger probe pulse oximetry (Ohmeda 3700e). From these results, a calibration curve was produced showing arterial oxygen saturation achieved with each different inspired oxygen concentration.

Oxygen was then delivered to either the ventilator tubing (fig. 1b) or the nasal mask (fig. 1c) at flow rates between 0 and $6 \mathrm{~L} \cdot \mathrm{min}^{-1}$. Oxygen saturation was again measured after 5 min at each flow rate, and by using the calibration curve for each individual patient, the inspired oxygen concentration received at each flow rate was estimated. 


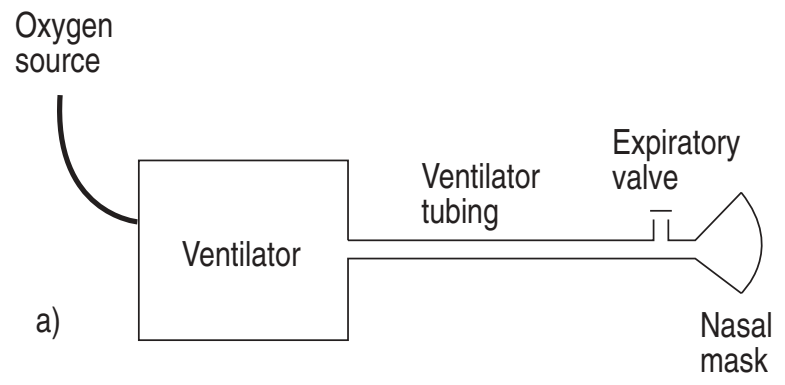

b)

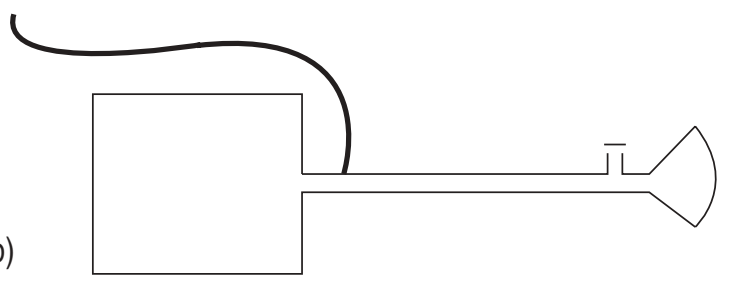

c)

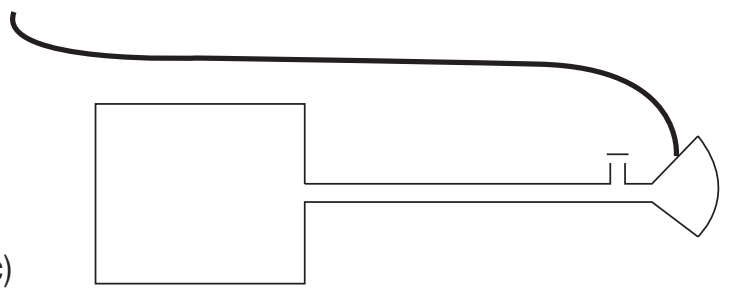

Fig. 1. - Routes by which supplemental oxygen can be supplied. a) through the ventilator; $b$ ) into the ventilator tubing, proximal to the expiratory valve; c) into the nasal mask, distal to the expiratory valve.

To investigate the changes in oxygen concentration received throughout the respiratory cycle, the sampling line of a polarographic oxygen analyser (Capnomac Ultima; Datex Inc.) was introduced into the minitracheostomy tube of one patient prior to the above experiment (the tube was already in situ for other reasons). Oxygen concentration was recorded throughout the respiratory cycle when supplemental oxygen was supplied at all flow rates by both routes.

Statistical analysis was performed using analysis of variance on the FASTAT computer statistical package.

\section{Results}

Figure 2 shows the results for all patients at all oxygen flow rates when oxygen was supplied by both routes. There is no significant difference between the oxygen concentration achieved by either route at any flow rate. Inspired oxygen concentration supplied by both routes rises nonlinearly with increases in oxygen flow rate, with little change at flows above $4 \mathrm{~L} \cdot \mathrm{min}^{-1}$. Although there is a wide scatter on each of the points, averaging the mean inspired oxygen concentration for both routes shows that: $1 \mathrm{~L} \cdot \mathrm{min}^{-1}$ oxygen supplies approximately $31 \%$ oxygen; $2 \mathrm{~L} \cdot \mathrm{min}^{-1}$ supplies $37 \%$; $3 \mathrm{~L} \cdot \mathrm{min}^{-1}$ supplies $40 \%$; 4 $\mathrm{L} \cdot \mathrm{min}^{-1}$ supplies $44 \%$; and 5 and $6 \mathrm{~L} \cdot \mathrm{min}^{-1}$ both supply $46 \%$.

Figure 3 shows the tracheal oxygen concentration throughout the respiratory cycle when oxygen was supplied either into the ventilator tubing or the nasal mask.

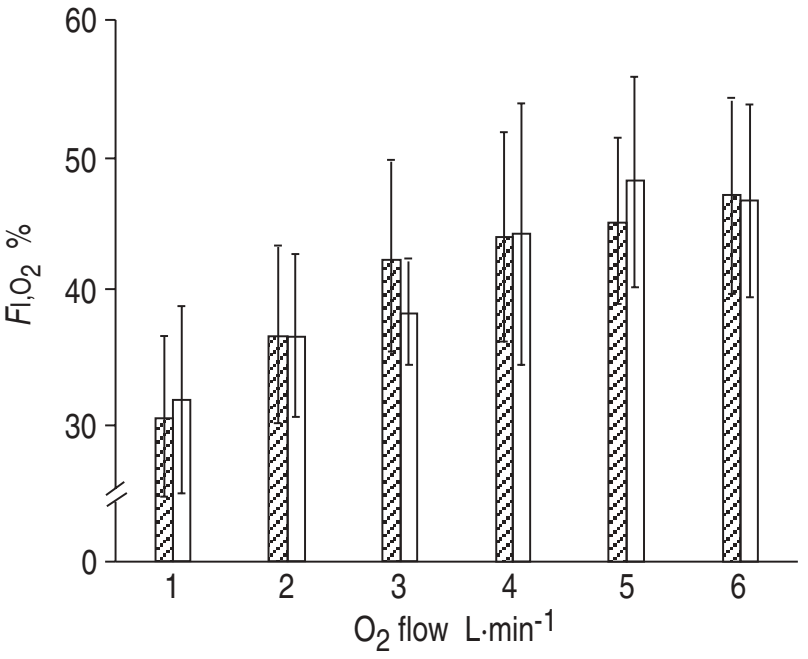

Fig. 2. - Inspired oxygen fraction $\left(F_{\mathrm{I}, \mathrm{O}_{2}}\right)$ received by the subjects at flow rates between 0 and $6 \mathrm{~L} \cdot \mathrm{min}^{-1}$ when oxygen is delivered into the nasal mask and into the ventilator tubing. Values are presented as mean \pm SD. $\square$ : mask; $\square$ : ventilator tubing.

\section{Discussion}

We have shown that a similar oxygen concentration is achieved by providing supplemental oxygen into the ventilator tubing or into the nasal mask (fig. 2). The wide scatter on each of these points is similar to that previously found in studies investigating supplemental oxygen supplied to spontaneously ventilating patients via variable performance masks [3] and nasal cannulae [4]. For variable performance masks, the scatter is due

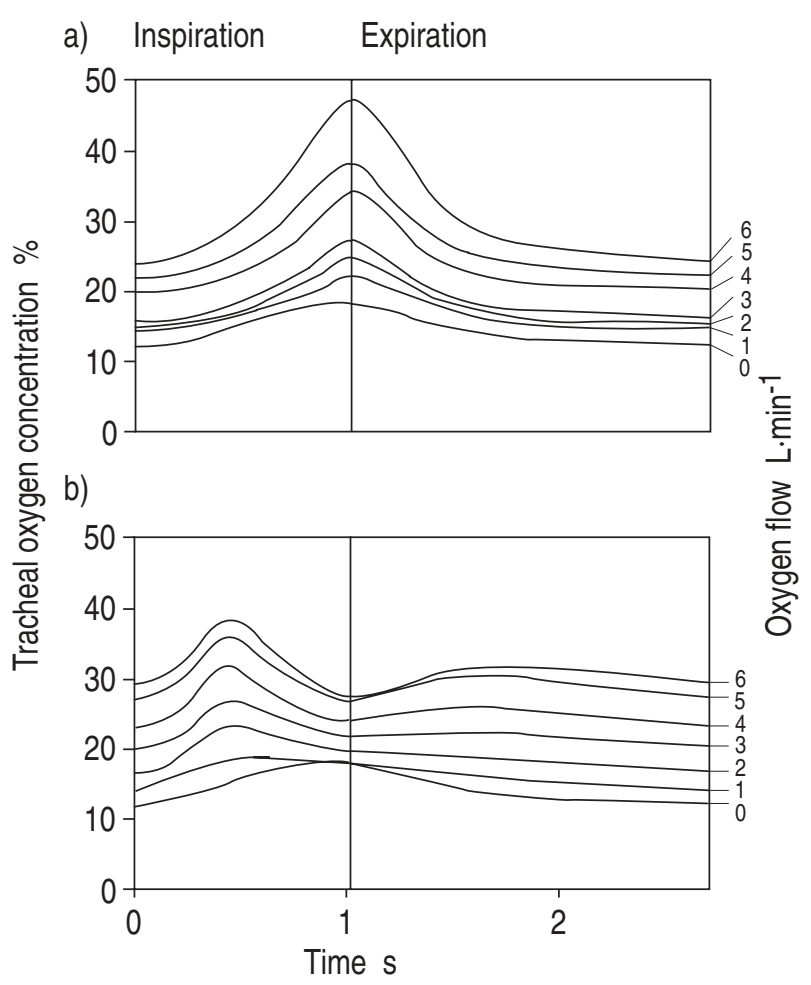

Fig. 3. - Tracheal oxygen concentration measured in one patient throughout the respiratory cycle when oxygen is delivered to: a) the ventilator tubing; or b) the nasal mask, at flow rates between 0 and 6 $\mathrm{L} \cdot \mathrm{min}^{-1}$. 
to differences in inspiratory flow between patients [3], and the different ventilator settings for each patient in this study is likely to have been the reason for the wide interindividual variation at each flow rate. The results, therefore, show the range of oxygen concentrations that may be seen with different ventilator settings in clinical practice.

When oxygen is supplied via the ventilator tubing (proximal to the expiratory valve) the patient initially receives anatomical dead space gas followed by mask dead space gas during inspiration, so that the tracheal oxygen concentration initially remains close to that of the end-expired gas (fig. 3a). The tracheal oxygen concentration then rises to a maximum at end-inspiration. This is due to gas from the ventilator tubing, which has acted as an oxygen reservoir during the previous expiration. The highest concentration of oxygen is, therefore, found to be delivered to the anatomical dead space.

When oxygen is supplied via the nasal mask (distal to the expiratory valve), the patient initially receives gas with an oxygen concentration close to the end-expiratory oxygen concentration (fig. 3b). This gas is from the anatomical dead space. Thereafter, the patient receives gas from the nasal mask, which has been enriched with oxygen during the previous expiratory phase and endexpiratory pause. In the latter parts of inspiration, the inspired oxygen concentration is that of the air in the ventilator tubing, supplemented with oxygen as it passes through the nasal mask. The highest concentration of oxygen is achieved in mid-inspiration, when gas is being delivered to the alveoli.

Despite these marked differences between the two routes in the time during inspiration at which the patient receives maximal supplemental oxygen, the total oxygen supply was similar with both routes, as seen in figure 2 .

The nonlinear increase in inspired oxygen concentration with increasing flow rate (fig. 2) may have been due to dead space gas when oxygen was supplied via the ventilator tubing. The oxygen concentration of dead space gas will be approximately equal to the end-expired concentration, which does not rise linearly with inspired oxygen concentration because of shunt. This effect would be expected most at higher inspired oxygen concentrations, as is seen.

When oxygen is supplied into the nasal mask, the mask dead space is effectively removed. At flow rates above $2 \mathrm{~L} \cdot \mathrm{min}^{-1}$, the oxygen concentration is lower in the endinspiratory gas than in expiratory (i.e. alveolar) gas (fig. 3 ). This implies that the late inspiratory ventilator tubing gas is limiting the maximum oxygen concentration available, and may be the cause of the nonlinearity seen in figure 2 when oxygen is supplied via the nasal mask.

In summary, oxygen supplementation during nasal intermittent positive pressure ventilation can be provided into the ventilator tubing or the nasal mask. Both these routes supply a similar amount of oxygen for each oxygen flow rate. The inspired oxygen concentration attained at each flow rate will vary considerably between patients, though as an approximate guide: $1 \mathrm{~L} \cdot \mathrm{min}^{-1}$ will provide $31 \% ; 2 \mathrm{~L} \cdot \mathrm{min}^{-1}$ will provide $37 \% ; 3 \mathrm{~L} \cdot \mathrm{min}^{-1}$ will provide $40 \%$; and $4 \mathrm{~L} \cdot \mathrm{min}^{-1}$ will provide $44 \%$. The maximum inspired oxygen concentration achievable by both routes is limited, and there is little benefit in increasing oxygen flow above $4 \mathrm{~L} \cdot \mathrm{min}^{-1}$.

\section{References}

1. Kinnear WJM. In: Assisted Ventilation at Home. Oxford, Oxford University Press, 1994.

2. Wysocki M, Tric L, Wolff MA, Gertner J, Millet H, Herman B. Noninvasive pressure support ventilation in patients with acute respiratory failure. Chest 1993; 103 : 907-913.

3. Leigh JM. Variation in performance of oxygen therapy devices. Ann $R$ Coll Surg Engl 1973; 52: 234-253.

4. Bazuaye EA, Stone TN, Corris PA, Gibson GJ. Variability of inspired oxygen concentration with nasal cannulas. Thorax 1992; 47: 609-611. 